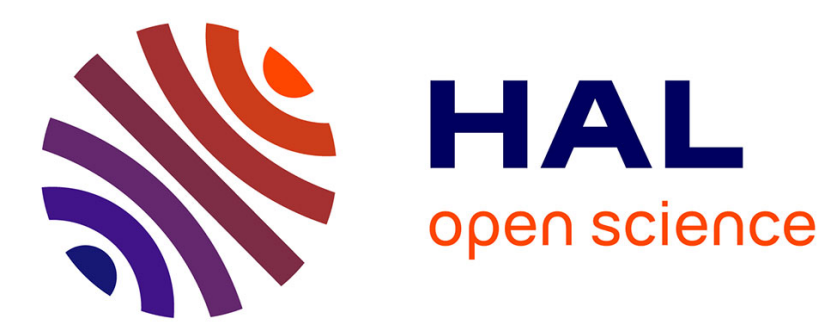

\title{
Parametric X-rays from ultrarelativistic electrons in a crystal : theory and possibilities of practical utilization
}

V.G. Baryshevsky, I.D. Feranchuk

\section{To cite this version:}

V.G. Baryshevsky, I.D. Feranchuk. Parametric X-rays from ultrarelativistic electrons in a crystal: theory and possibilities of practical utilization. Journal de Physique, 1983, 44 (8), pp.913-922. 10.1051/jphys:01983004408091300 . jpa-00209674

\section{HAL Id: jpa-00209674 https://hal.science/jpa-00209674}

Submitted on 1 Jan 1983

HAL is a multi-disciplinary open access archive for the deposit and dissemination of scientific research documents, whether they are published or not. The documents may come from teaching and research institutions in France or abroad, or from public or private research centers.
L'archive ouverte pluridisciplinaire HAL, est destinée au dépôt et à la diffusion de documents scientifiques de niveau recherche, publiés ou non, émanant des établissements d'enseignement et de recherche français ou étrangers, des laboratoires publics ou privés. 
Classification

Physics Abstracts

$07.85-29.90$

\title{
Parametric X-rays from ultrarelativistic electrons in a crystal : theory and possibilities of practical utilization
}

\author{
V. G. Baryshevsky and I. D. Feranchuk \\ Department of Physics, Byelorussian State University, Minsk-80, USSR
}

(Reçu le 16 août 1982, première révision le 21 décembre 1982, deuxième révision le 15 mars 1983, accepté le 26 avril 1983)

\begin{abstract}
Résumé. - Dans cet article on présente la théorie détaillée de l'émission de rayons $\mathrm{X}$ lors du passage de particules chargées dans les cristaux. On montre que cette émission provient de la diffraction du champ électromagnétique propre de la particule et possède des particularités spécifiques qui permettent de la distinguer des autres mécanismes d'émission. Les méthodes expérimentales utilisées dans l'étude de l'émission paramétrique des rayons $\mathrm{X}$ sont discutées également.
\end{abstract}

\begin{abstract}
The detailed theory of parametric X-rays from charged particles in a crystal is considered. It is shown that this radiation is due to the diffraction of the particle's electromagnetic field. Some features of the parametric $\mathrm{X}$-rays allow us to separate this radiation from other radiation types. Methods of the experimental investigation and uses of parametric $\mathrm{X}$-rays are discussed.
\end{abstract}

\section{Introduction.}

At present the problem of obtaining intense and monochromatic X-ray beams is of great interest. The proposed solution to this problem involves electromagnetic radiation from relativistic charged particles in external fields in various media. Theoretical and experimental investigations of many radiation types including synchrotron [1] and undulator [2] radiation, bremsstrahlung [3] and channelling radiation [4, 5] have been carried out. However, the type of radiation considered here possesses some useful features but has as yet been described theoretically only.

We refer to the parametric X-rays (PX) which originate when a relativistic charged particle interacts with a crystal. This radiation is due to the periodic character of the crystal's dielectric constant. The optical parametric radiation was first described by Fainberg and Thiznjak [6]. They considered the radiation from a uniformly moving electron in a medium with a simply periodic dielectric constant. Analogous radiation in a thin triply periodic crystal was considered by Ter-Mikaeljan [7]. But true PX occurs in a crystal with thickness $L$, greater than the $\mathrm{X}$-ray extinction length, which is

$$
K L|n-1| \gtrsim 1,
$$

where $K$ is the emitted photon wave number and $n$ is the crystal refraction index; $\hbar=c=1$.
In this case the emitted photon state in the crystal is modified in an essential way. The importance of such a modification was first mentioned by Baryshevsky [8]. In subsequent articles we presented the classical [9] and quantum [10] theories of PX. The classical theory of this phenomenon was independently considered by Garibjan and Jan Shee [11]. Later several theoretical papers were published where the same radiation mechanism was described [12, 13].

We do not know of any experimental investigations on PX despite the fact that this phenomenon was predicted quite long ago and several possible uses were described $[14,15]$. This could be connected with the lack of theoretical papers with a simple and clear description of the features inherent to PX which distinguish this radiation from other radiation types.

The present paper is an attempt to fill in this gap in order to call attention to the discovery and investigation of PX. This radiation may be used both as an X-ray source with a number of useful properties and for obtaining information about crystal structure.

\section{Qualitative consideration.}

Let us suppose that an ultrarelativistic electron $(E \gg m)$, enters a crystal. Here $E$ is the energy and $m$ is the mass of the particle. The crystal is arbitrarily oriented with respect to the electron velocity $\mathbf{v}$. In order to give a simple analysis of the PX for- 
mation we recall that the relativistic charged particle's electromagnetic field can be represented as a superposition of pseudophotons whose properties are close to those of real photons [16]. The pseudophotons have angular spread $\Delta \theta \lesssim m / E$ and their spectral distribution is determined as follows [16]

$$
n(\omega)=\frac{2}{\pi \omega} \ln \left(\eta \frac{E}{\omega}\right) ; \quad \omega \ll E,
$$

where $\omega$ is the pseudophoton frequency and $\eta$ has a value of the order of unity.

From this point of view, the electromagnetic interaction of the ultrarelativistic electron with the crystal is equivalent to the interaction of a photon beam (angular spread $\Delta \theta$ and spectrum I) with the crystal (Fig. 1). PX can then be considered to be the result of X-pseudophoton diffraction in the crystal. The pseudophoton diffraction as well as the diffraction of external X-rays is a coherent process, i.e. the probability of this process is in direct proportion to the square of the crystal length. Therefore under certain fixed conditions the PX intensity may essentially exceed the intensity of the pseudophoton incoherent scattering which leads to bremsstrahlung [16].

Because of the pseudophoton diffraction the PX travels both along the direction of the electron velocity and at large angles relative to it. These angles are determined by the crystal reciprocal lattice vectors (for example, the parametric radiation in the - $\mathbf{v}$ direction is possible). This angular distribution is the most important feature inherent to PX which enables us to separate this radiation from other secondary processes due to the ultrarelativistic particle interaction with the crystal. The point is that the particle formed in the secondary processes (photons, electron-positron pairs and so on) leave the crystal at small angles $(\sim m / E)$ relative to $\mathbf{v}$.

In order to consider the interaction of the pseudophotons with the crystal one can use the results of the theory of the diffraction of X-rays and resonant $\gamma$-radiation [18]. In the case $E \gg m$ the pseudophoton momentum can be written as

$$
\mathbf{K}=\omega \frac{\mathbf{v}}{v^{2}} \approx \omega \mathbf{v}
$$

Those pseudophotons whose momenta satisfy the

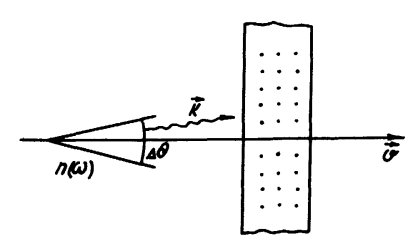

Fig. 1. - Diagram of the spectral and angular spread of pseudophotons.
Bragg condition

$$
(\mathrm{K}+\tau)^{2} \approx K^{2},
$$

are diffracted by the crystal. Such a process leads to parametric $X$-radiation in the $(K+\tau)$ direction. Essentially, the photons are emitted from the crystal at an angle which does not depend on the energy $E$. This angle is defined by the particle's velocity orientation relative to the crystallographic plane (Fig. 2). Here $\tau$ is the reciprocal lattice vector of the crystal.

Depending on $\tau$, the emitted photons propagate both at an angle smaller than $\pi / 2$ relative to $\mathbf{v}$ (the Laue case) and at an angle greater than $\pi / 2$ (the Bragg case). The pseudophoton spectrum defined by equation (1) is continuous and therefore the condition is fulfilled for all reciprocal lattice vectors simultaneously. The intensity of PX in the direction of $(\mathbf{K}+\tau)$ depends on the structure factor $F(\tau)$ determining the probability of $\mathrm{X}$-ray coherent scattering with momentum transfer $\tau$. An analogous situation takes place when a polychromatic X-ray beam is diffracted by a crystal.

The pseudophotons have an angular spread $\Delta \theta \sim m / E$ [16]. As a consequence PX propagates in the direction of $\mathbf{K}+\tau \approx \omega \mathbf{v}+\tau$ in a cone with angular divergence $\Delta \theta$. Therefore, lateral spots have to appear on the X-film placed to the right or to the left of the crystal. The distribution of these spots does not depend on the particle energy and coincides with the totality of reflexes formed when an X-ray beam with spectrum (I) is diffracted by the crystal. These lateral X-ray peaks from ultrarelativistic charged particles are the experimental evidence of the PX formation.

It should be noted that pseudophoton coherent diffraction is not a unique mechanism of the PX formation. Analogous radiation arises as a result of bremsstrahlung photon diffraction as well as the diffraction of photons emitted by a channelled charged particle [4]. But the pseudophoton coherent diffraction mechanism of PX formation is of importance for the quantitative calculation of PX intensity (see below) and does not affect the lateral spot distribution.

Qualitative analysis based on the consideration of the pseudophoton diffraction enables us to find both angular and spectral distribution of PX. In fact, coherent photon emission in the direction $(\omega \mathbf{v}+\tau)$ is possible if the condition (2) is fulfilled.

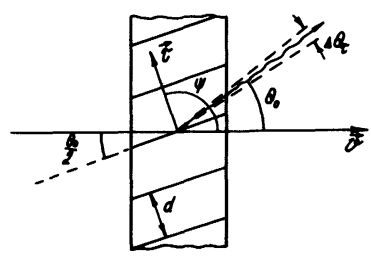

Fig. 2. - Geometry of an experiment to observe the parametric X-rays. 
This photon frequency is calculated as follows

$$
\omega_{\tau}=\frac{\tau^{2}}{2|(\tau . v)|} \quad \text { or } \quad \omega_{\tau}=\frac{\tau}{2|\cos \psi|}=\frac{\pi n}{\mathrm{~d}|\cos \psi|},
$$

where $\psi$ is the angle between $\mathbf{v}$ and a crystallographic plane corresponding to the vector $\tau$ (Fig. 2).

It follows from equation (4) that because of the pseudophoton angular spread $\Delta \theta$, a lateral spot corresponds to a quasimonochromatic $\mathrm{X}$-ray beam with spectral spread

$$
\frac{\Delta \omega}{\omega_{\tau}} \approx \frac{m}{E}
$$

Crystal periodicity also changes the spectrum of the photons emitted along the particle velocity direction, in comparison with a homogeneous medium [15]. The point is that in the crystal the coherent scattering of the pseudophotons with frequency $\omega_{\tau}$ leads to an X-ray beam forming along the vector $\omega \mathbf{v}+\tau$. The amplitude a wave travelling along the vector $\mathbf{v}$ also changes. As a consequence the spectrum of photons emitted along the particle velocity direction changes within the interval defined by the conditions (4) and (5). We shall now consider the detailed characteristics of PX.

\section{Quantum theory of the parametric X-rays.}

The main difficulty in describing PX is connected with the fact that perturbation theory fails to take into account both particle and emitted photon interaction with a thick monocrystal. Therefore in reference [10] the use of quantum electrodynamics in Furry notation [16] for describing electromagnetic processes in the crystal was suggested. It was shown that the quantum calculation was simpler in the case when both quantum and classical considerations were applied. According to reference [10] any electromagnetic process in a thick crystal is defined by the same Feynman diagram as for this process in quantum electrodynamics, but in the crystal each line on this diagram corresponds not to a plane wave but to an accurate stationary wave function of a particle or photon. We shall picture these states by means of double lines on the diagrams. For example, to first order in the fine structure constant, the radiation process (an electron with energy $E$ is in the initial state while an electron with energy $E_{1}$ and a photon with energy $\omega$ are in the final state) is defined by the diagram on figure $3 a$. This diagram corresponds to the following analytical expression

$$
\begin{aligned}
M_{\mathrm{fi}}=2 \pi e \delta\left(E-E_{1}\right. & -\omega) \times \\
& \times \int\left(\varphi_{E_{1}}^{-}\right)^{*} \gamma_{\mu}\left(\mathcal{A}_{\mu}^{-}\right)^{*} \varphi_{E}^{+} \mathrm{dr} .
\end{aligned}
$$

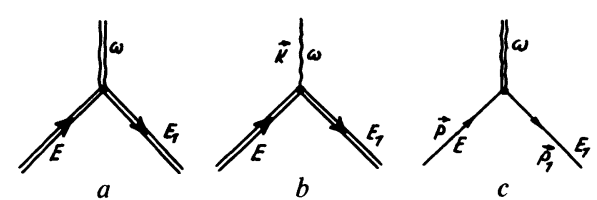

Fig. 3. - Feynman diagrams for electromagnetic radiation from electrons in the crystal.

Here $\varphi_{E}^{+}\left(\varphi_{E_{1}}^{-}\right)$is the electron stationary wave function which consists of the superposition of plane and outgoing (ingoing) spherical waves in the limit $|\mathbf{r}| \rightarrow \infty . \mathcal{A}_{\mu}^{-}(\mathbf{r})$ is the stationary solution of Maxwell's equations which includes the ingoing spherical wave; $\gamma_{\mu}$ are the Dirac matrices.

It is well known [16] that, because of the momentum and energy conservation laws, the matrix element (6) is equal to zero identically when the radiation process takes place in vacuum. But in the crystal, the electron and photon wave functions are changed in comparison with those in vacuum and therefore the quantity $M_{\mathrm{fi}}$ is nonzero. This matrix element makes the main contribution to the radiation cross section.

In order to use expression (6) in calculations one has to find the particle wave functions $\varphi_{E}^{ \pm}$and the vector potential $\mathcal{A}_{\mu}^{-}$. Obviously, exact functions $\varphi_{E}^{ \pm}$ cannot be found but one can usually choose an approximation method which takes into account the main physical processes involved in the experiment. There are two main radiation channels for ultrarelativistic particles in a crystal. Figures $3 b, c$ show the diagrams corresponding to these channels.

Double lines on the diagram $3 b$ correspond to the channelled particles wave functions and in this case the matrix element (6) corresponds to the channelling radiation [4]. This diagram may be used when the emitted photon refraction and diffraction are not essential. Diagram $3 c$ defines the parametric X-rays when the photon is diffracted and described by the function $\mathcal{A}_{\mu}^{-}(\mathbf{r})$, with the particle moving at uniform velocity. The radiation cross section for the general diagram (Fig. 3a) was calculated in references [4, 10]. In reference [10] the particle wave function was considered in the eikonal approximation and in reference [4] the channelled particle wave function was used. It was shown in paper [17] that the PX intensity in the peak essentially exceeds the channelling radiation intensity and is described by the following matrix element

$$
\begin{aligned}
& M_{\mathrm{fi}}^{(0)}=2 \pi e \delta\left(E-E_{1}-\omega\right) \times \\
& \quad \times \int \mathrm{e}^{i\left(\mathbf{p}-\mathbf{p}_{1}\right) \mathbf{r}} \mathbf{U}_{\mathbf{p}_{1}} \gamma_{\mu} u_{\mathbf{p}}\left(\mathcal{A}_{\mu}^{-}\right)^{*} \mathrm{~d} \mathbf{r} \equiv \\
& \equiv T_{\mathrm{fi}}^{(0)} \delta\left(E-E_{1}-\omega\right),
\end{aligned}
$$

where $\mathbf{p}\left(\mathbf{p}_{1}\right)$ is the particle momentum in the initial (final) state; $u_{p}$ is the spinor corresponding to a free particle with momentum $\mathbf{p}$. 
Essentially, the value $M_{\mathrm{fi}}^{(0)}$ contains the solution of the homogeneous Maxwell equations only and this enables us to simplify the calculations in comparison with the classical consideration of this problem [11]. The coherent interaction of the photon with a crystal can be taken into account via the crystal dielectric constant. This approach is a result of the Maxwell equations being averaged over the crystal state [18]. Because of the translation symmetry the dielectric constant tensor is

$$
\chi_{i j}\left(\mathbf{r}, \mathbf{r}^{\prime}, t-t^{\prime}\right)=\int \mathrm{d} \mathbf{K} \mathrm{d} \omega \sum_{\tau} \chi_{i j}\left(\mathbf{K}, \mathbf{K}_{\tau}, \omega\right) . \exp \left\{i\left[\mathbf{K}\left(\mathbf{r}-\mathbf{r}^{\prime}\right)-\tau \tau^{\prime}-\omega\left(t-t^{\prime}\right)\right]\right\},
$$

where $\mathbf{K}_{\tau}=\mathbf{K}+\tau$ and quantities $\chi_{i j}(\omega)$ are directly connected with the amplitude of the photon coherent scattering by the electrons and nuclei of the crystal. The obvious form of these expressions was found in the theory of $\mathrm{X}$ - and gamma-ray diffraction $[18,19]$ and is

with

$$
\chi_{i j}\left(\mathbf{K}, \mathbf{K}_{\tau}, \omega\right)=\delta_{i j} \delta_{\mathbf{K} \mathbf{K}_{\tau}}+\chi_{i j}^{(1)}\left(\mathbf{K}, \mathbf{K}_{\tau}, \omega\right)
$$

$$
\chi_{i j}^{(1)}=\frac{4 \pi}{\omega^{2} \Omega_{0}} \sum_{n} \mathrm{e}^{i \tau \mathbf{R}_{n}}\left(f_{i j n}^{(\mathbf{e})}+f_{i j n}^{(\mathbf{n})}\right) .
$$

Here, $\Omega_{0}$ is the unit cell volume; $R_{n}$ is the coordinate of the $n$-th atom in the cell; $f_{i j n}^{(\mathrm{e})}$ and $f_{i j n}^{(\mathrm{n})}$ are the amplitudes of the photon coherent scattering by the atomic electrons and nucleus, respectively. These amplitudes are defined as follows :

$$
f_{i j n}^{(\mathrm{e})}=-r_{0} \delta_{i j} \mathrm{e}^{-\frac{1}{2} Z(\tau)}\left\{F_{n}(\tau)+\Delta f^{\prime}(\omega)+i \Delta f^{\prime \prime}(\omega)\right\}
$$

Here, $F_{n}(\tau)$ is the atomic form factor; $\Delta f^{\prime}$ and $\Delta f^{\prime \prime}$ are the anomalous dispersion corrections [19]; $r_{0}=e^{2} / m$; $\mathrm{e}^{-\frac{1}{2} z}$ is the Debye-Waller factor. The amplitude $f_{i j n}^{(n)}$ is [18]

$$
f_{i j n}^{(\mathbf{n})}\left(\mathbf{K}, \mathbf{K}_{\tau}\right)=-\frac{1}{4 \omega_{r}} \cdot \frac{2 J_{1}+1}{\left(2 J_{0}+1\right)} \cdot \frac{F_{1}}{\left(\omega-\omega_{1}+i \frac{\Gamma}{2}\right)} \cdot \mathfrak{T}_{i j}\left(\mathbf{K}, \mathbf{K}_{\tau}\right) \mathcal{Q}_{n}
$$

with

$$
\begin{aligned}
& \mathfrak{T}_{i j}=\frac{\left(\mathbf{K K}_{\tau}\right) \delta_{i j}-K_{\tau j} K_{i}}{\omega_{r}^{2}} \text { for } \mathbf{M} 1 \text { transitions } \\
& \mathfrak{T}_{i j}=\frac{1}{\omega_{r}^{2}}\left[\left(\mathbf{K K}_{\tau}\right) \delta_{i j}+K_{\tau i} K_{\tau j}-2 K_{i} K_{\tau j}\right] \text { for E2 transitions } \\
& \mathcal{Q}_{n}=\left\{\begin{array}{c}
\exp \left[-\frac{1}{2} Z_{n}(\mathbf{K})-\frac{1}{2} Z_{n}\left(\mathbf{K}_{\tau}\right)\right] \text { if } \Gamma \ll \omega_{\mathrm{ph}} \\
\exp \left[-\frac{1}{2} Z_{n}(\tau)\right] \text { if } \Gamma \gg \omega_{\mathrm{ph}} ; \\
Z_{n}(\mathbf{K})=K^{2}{\overline{\mathcal{U}_{n}}}_{n}^{2} .
\end{array}\right.
\end{aligned}
$$

Further, $\omega_{r}, \Gamma_{1}$ and $\Gamma$ are the frequency, the elastic and the total widths of the resonant level, respectively; $J_{1}\left(J_{0}\right)$ is the angular momentum of the excited (ground) nucleus state; $\omega_{\mathrm{ph}}$ is the characteristic frequency of the crystal phonon spectrum; and $\mathcal{U}_{n}$ is the atom oscillation amplitude.

The Maxwell equations with dielectric constant (8-9) can be solved in the two-beam approximation with an accuracy of $\left\|\chi_{i j}^{(1)}\right\| \sim 10^{-4} \div 10^{-5}$ in the $\mathrm{X}$-ray region $[18,19]$. The solution of these equations was found in X-ray diffraction theory for the vector potential $\mathcal{A}_{\mu}^{+}(\mathbf{r})$ which asymptotically consists of the plane and outgoing spherical waves. But, just as the amplitude of any process occurring when the photon is created inside the crystal, the matrix element (7) contains the solution $\mathcal{A}_{\mu}^{-}$with a different asymptotic behaviour. But it is not necessary to solve the Maxwell equations once again because of the relationship [10]

$$
\mathcal{A}_{\mu}^{-}(\mathbf{K}, \mathbf{r})=\left[\mathcal{A}_{\mu}^{+}(-\mathbf{K}, \mathbf{r})\right]^{*} .
$$


This formula allows us to obtain the function $\mathcal{A}_{\mu}^{-}$using the solution $\mathcal{A}_{\mu}^{+}$. Figure 4 shows the primary and diffracted waves corresponding to $\mathcal{A}_{\mu}^{-}$in the Laue and Bragg cases. We give the form of $\mathcal{A}_{\mu}^{-}(\mathbf{r})$ in the Laue case only :

$$
\begin{aligned}
& \mathcal{A}_{\mathbf{K s}}^{-}(\mathbf{r})=\left[\mathcal{A}_{s}(\boldsymbol{\rho}, 0)+\mathcal{A}_{1 s}(\rho, 0) \mathrm{e}^{i \tau_{Z} Z} \mathrm{e}^{i K_{Z} Z}\right] \theta(-Z)+\left[\mathcal{A}_{s}(\mathbf{r})+\mathcal{A}_{1 s}(\mathbf{r})\right] \theta_{r} \theta(L-Z)+\mathbf{e}_{s} \cdot \mathrm{e}^{i \mathbf{K r}} \theta(Z-L), \\
& \theta(Z)=\left\{\begin{array}{l}
1, Z>0 \\
0, Z<0
\end{array}\right. \\
& \boldsymbol{A}_{s}(\mathbf{r})=\mathbf{e}_{s} \frac{\mathrm{e}^{i \mathbf{R r}}}{2\left(\varepsilon_{2 s}^{*}-\varepsilon_{1 s}^{*}\right)} \sum_{\lambda=1,2}\left(2 \varepsilon_{\lambda s}^{*}-g_{00}\right) \exp \left[-i \frac{\omega}{\gamma_{0}} \varepsilon_{\lambda s}^{*}(L-Z)\right] \\
& \boldsymbol{t}_{1 s}(\mathbf{r})=-\mathbf{e}_{\tau s} g_{01}^{(s) *} \frac{\mathrm{e}^{i \mathbf{K}_{\tau} \mathbf{r}}}{2\left(\varepsilon_{2 s}^{*}-\varepsilon_{1 s}^{*}\right)} \sum_{\lambda} \exp \left[-i \frac{\omega}{\gamma_{0}} \varepsilon_{\lambda s}^{*}(L-Z)\right]
\end{aligned}
$$

where

$$
\begin{gathered}
\varepsilon_{\lambda s}=\frac{a}{4} \pm \frac{1}{4}\left[a^{2}+4 \beta g_{00} \alpha-g_{00} g_{11}+g_{10}^{(s)} g_{01}^{(s)}\right]^{1 / 2} \\
a=g_{00}+\beta g_{11}-\beta \alpha ; \quad \gamma_{0}=\cos (\mathbf{K v}) ; \quad \beta=\frac{K_{Z}}{K_{Z}+\tau_{Z}} ; \quad \lambda, s=1,2 .
\end{gathered}
$$

The index $s$ means the polarization and $\lambda$ means $( \pm)$ sign before the square root in formula (15).

$\alpha=\left(2 \mathbf{K} \tau+\dot{\tau}^{2}\right) \omega^{-2}$ is a value characterizing the divergence of $\mathbf{K}$ from the Bragg condition (3); $\mathbf{e}_{1} \|[\mathbf{K} \tau]$; $\mathbf{e}_{2}\left\|\left[\mathbf{K e}_{1}\right] ; \mathbf{e}_{\tau 1}\right\| \mathbf{e}_{1} ; \mathbf{e}_{\tau 2} \|\left[\mathbf{K}_{\tau} \mathbf{e}_{\tau 1}\right]$, and the values $g_{\alpha \beta}^{(s)}$ are defined as follows

$$
\begin{array}{ll}
g_{00}=e_{1 i} e_{1 j} \chi_{i j}^{(1)}(\mathbf{K} ; \mathbf{K}) ; & g_{11}=e_{\tau 1 i} e_{\tau 1 j} \chi_{i j}^{(1)}\left(\mathbf{K}_{\tau}, \mathbf{K}_{\tau}\right) \\
g_{01}^{(s)}=e_{s i} e_{\tau s j} \chi_{i j}^{(1)}\left(\mathbf{K}, \mathbf{K}_{\tau}\right) ; & g_{10}^{(s)}=e_{\tau s i} e_{s j} \chi_{i j}^{(1)}\left(\mathbf{K}_{\tau}, \mathbf{K}\right) .
\end{array}
$$

In those cases where the tensor $f_{i j}^{(\mathbf{n})}$ is more complicated than that of equation(11), it is convenient to describe the functions $\mathcal{A}_{\mu}^{-}$by means of the covariant method considered in reference [20]. The matrix element of equation (7) defines the number of photons which are emitted by an electron in the crystal :

$$
\frac{\partial^{2} N}{\partial \omega \partial \Omega}=J \omega^{2} \sum \int\left|T_{f i}^{(0)}\right|^{2} \delta\left(E-E_{1}-\omega\right) \frac{\mathrm{d} \mathbf{p}_{1}}{(2 \pi)^{6}}
$$

where $\sum$ means an averaging over the initial polarization states and summation over the final ones; $\mathfrak{J}$ is the number of electrons entering into the crystal per unit time. Using equations $(13,14)$ in equations $(7,17)$ one obtains the following result $(\omega \ll E)$ :

$$
\frac{\partial^{2} N_{s}^{\tau}}{\partial \omega \partial \Omega_{\tau}}=\frac{e^{2}}{4 \pi \omega} \mathfrak{J}\left|\sum_{\lambda=1,2}\left(\mathbf{e}_{\tau s} \mathbf{v}_{\tau}\right) B_{\lambda s}^{\tau}\left[\exp \left(-i \omega L q_{\lambda s}^{\tau}\right)-1\right]\right|^{2}
$$

where $\partial^{2} N_{s}^{\tau}$ is the number of photons with polarization $\mathbf{e}_{\tau s}$ emitted within the spectral interval $\mathrm{d} \omega$ and with angular spread $\mathrm{d} \Omega_{\tau}$; the angle is counted off the vector $(\omega \mathbf{v}+\tau)$; indices $s=1,2$ correspond to the photon being polarized in the plane of the vectors $\mathbf{v}$ and $\tau$ and in the perpendicular direction, respectively. For the Laue case one finds

$$
B_{\lambda s}^{\tau}=\gamma_{\lambda s}^{\tau}\left(\frac{1}{q_{0}^{\tau}}-\frac{1}{q_{\lambda s}^{\tau}}\right)
$$

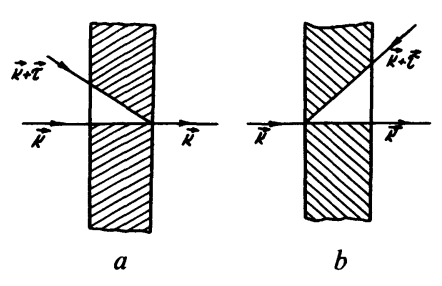

Fig. 4. - Diagrams of spreading of forward and diffracted waves in the function $\left.A_{\mu}^{-}(\mathbf{r}): a\right)$ Laue case; $b$ ) Bragg case. 
and

$$
\begin{array}{ccc}
\gamma_{\lambda s}^{\tau}=(-1)^{\lambda} \frac{2 \varepsilon_{\lambda s}-g_{00}}{2\left(\varepsilon_{2 s}-\varepsilon_{1 s}\right)} & \text { if } & \tau=0 \\
\gamma_{\lambda s}^{\tau}=(-1)^{\lambda} \frac{g_{01}^{(s)}}{2\left(\varepsilon_{2 s}-\varepsilon_{1 s}\right)} \quad \text { if } & \tau \neq 0 \\
q_{0 \tau}=1-v \cos \theta_{\tau} ; \quad g_{\lambda s}^{\tau}=g_{0}^{\tau}-\varepsilon_{\lambda s} ; & \mathbf{v}_{\tau}=\mathbf{v}+\frac{\tau}{\omega}
\end{array}
$$

where $\theta_{\tau}$ is the angle between the vectors $\mathbf{K}$ and $\omega \mathbf{v}+\tau$.

The quantities $\omega q_{0}^{\tau}$ and $\omega q_{\lambda s}^{\tau}$ are $Z$-axis projections of the momenta transferred to the vacuum and crystal, respectively, due to photon emission in the direction $(\omega \mathbf{v}+\tau)$ (the case $\tau=0$ is included). Thus the quantities $L_{0}^{\tau}=\left(\omega q_{0}^{\tau}\right)^{-1}$ and $L_{\lambda s}^{\tau}=\left(\omega q_{\lambda s}^{\tau}\right)^{-1}$ are the coherent radiation length [10], that is, those portions of the particle trajectory in the vacuum and in the crystal which give coherent radiation. The radiation intensity is proportional to the square of the coherence length and, therefore, the maximum number of photons is emitted in those directions where $L_{0}^{\tau}$ and $L_{\lambda s}^{\tau}$ are maximum. This takes place within the angular spread

$$
\Delta \theta_{\tau} \lesssim\left[\left(\frac{m}{E}\right)^{2}+\left|g_{00}\right|\right]^{1 / 2} .
$$

Unlike the homogeneous medium, in the crystal condition (20) is fulfilled not only for the photons emitted forward but also for the photons propagating along the vector $(\omega \mathbf{v}+\tau)$, directed at an angle $\theta_{0}$ relative to $\mathbf{v}$. Here $\theta_{0}$ is defined as follows (see Fig. 2)

$$
\cos \theta_{0}=\frac{\tau_{\perp}^{2}-\tau_{Z}^{2}}{\tau^{2}}
$$

This result coincides with the qualitative consideration of paragraph 2 .

Let us consider now the radiation spectrum. It is well known that the maximum value of the vacuum coherence length is defined by the expression [7]

$$
L_{0}^{\max }=\frac{2 E^{2}}{m^{2} \omega}
$$

At the same time it turns out that, in the crystal, there exists such a radiation angle where one of the quantities $\operatorname{Re} q_{\lambda s}^{\tau}$ vanishes. This means that, by neglecting absorption, the coherent length goes to infinity and radiation intensity increases as the square of the whole crystal dimension, just as it does for Vavilov-Cherenkov optical radiation in a homogeneous medium [7] and for channelling radiation [4]. Thus, the equation

$$
\operatorname{Re} q_{\lambda s}^{\tau}=1-v \cos \theta_{\tau}-\operatorname{Re} \varepsilon_{\lambda s}=0,
$$

constitutes the dispersion equation of the parametric X-rays in the crystal. In order to simplify further investigation let us consider a non-Mössbauer crystal when we do not need to take into account the resonant amplitude in equation (9). In this case the relations

$$
g_{\alpha \beta}^{\prime}<0 ; \quad g_{\alpha \beta}^{\prime} \gg g_{\alpha \beta}^{\prime \prime} ; \quad g_{00}=g_{11} ; \quad g_{\alpha \beta}=g_{\alpha \beta}^{\prime}+i g_{\alpha \beta}^{\prime \prime}
$$

are satisfied, and equation (23) in the Laue case $(\beta>0)$ has the form

$$
\gamma_{\tau}^{2}+x \mp \sqrt{\left(x+2 g_{00}^{\prime}\right)^{2}+4 \beta r_{s}^{\prime}}=0 .
$$

Here,

$$
\begin{gathered}
\gamma_{\tau}^{2}=2\left(\theta_{\tau}^{2}+\frac{m^{2}}{E^{2}}\right) ; \quad x=\beta \alpha-g_{00}^{\prime}-\beta g_{11}^{\prime} ; \quad r_{s}=g_{10}^{(s)} g_{01}^{(s)} \\
\alpha=\left[2 \omega\left(\tau_{\perp} \sin \theta_{\tau} \cos \varphi_{\tau}+\tau_{z} \cos \theta_{\tau}\right)+\tau^{2}\right] \omega^{-2}
\end{gathered}
$$

and $\varphi_{\tau}$ is the angle between $\mathbf{K}$ and $\tau$ in the plane, perpendicular to the vector $\omega \mathbf{v}+\tau$.

In the considered case, equation (25) with the negative root can be satisfied and the solution is

$$
x=\frac{4 g_{00}^{\prime 2}+4 \beta r_{s}^{\prime}-\gamma_{\tau}^{4}}{2\left(\gamma_{\tau}^{2}-2 g_{00}^{\prime}\right)}
$$


The imaginary part of the quantity $q_{1 s}^{\tau}$ is equal to

$$
\operatorname{Im} q_{1 s}^{\tau}=-\frac{2 \beta g_{00}^{\prime \prime}}{\left[\left(\gamma_{\tau}^{2}-2 g_{00}^{\prime}\right)^{2}+4 \beta r_{s}^{\prime}\right]}\left\{\left[\gamma_{\tau}^{2}-2 g_{00}^{1}+\frac{r_{s}^{\prime \prime}}{g_{00}^{\prime \prime}}\right]^{2}+4 r_{s}^{\prime}-\left(\frac{r_{s}^{\prime \prime}}{g_{00}^{\prime \prime}}\right)^{2}\right\} .
$$

When the Cherenkov condition (23) is fulfilled, the radiation intensity has its maximum proportional to the smaller of the two values $\omega^{2} L^{2}$ and $\left(q_{1 s}^{\prime \prime}\right)^{-2}$. The spectral intensity of PX at maximum is either $\left(g_{00}^{\prime} \omega L\right)^{2}$ or $g_{00}^{\prime 2} / g_{00}^{\prime \prime 2}$ times larger than the intensity of the transition radiation. Note that Cherenkov X-rays in a homogeneous medium are possible only when the radiation frequency is close to the resonance frequency of the atoms or nuclei [21]. But in this case $\left|g_{00}^{\prime}\right| \sim\left|g_{00}^{\prime \prime}\right|$ and the intensity of Cherenkov radiation is of the same order as the transition radiation intensity [21]. It is also important that the coherence length in the crystal be larger than the absorption length in the homogeneous medium. The point is that the Borrmann effect [19] for X-rays and the Kagan-Afanas'ev effect [18] for resonant $\gamma$-rays also arises in the radiation processes. So, if the emitted photon has a polarization perpendicular to the diffraction plane then the following inequality holds

$$
\Delta^{\prime \prime}=\operatorname{Im}\left(g_{00}^{2}-g_{01}^{(1)} g_{10}^{(1)}\right) \ll \operatorname{Im} g_{00}^{2}
$$

and the coherence length

$$
L_{11}^{\tau} \approx-\frac{\left(\gamma_{\tau}^{2}-2 g_{00}^{\prime}\right)^{2}+4 \beta r_{1}^{\prime}}{2 \omega \beta g_{00}^{\prime \prime} \gamma_{\tau}^{4}}
$$

increases with energy $\sim E^{4}$, unlike to the homogeneous medium, where it does not depend on $E$ (« density effect » [7]).

We emphasize once more that the collimated beams of quasimonochromatic X-rays, directed at large angles relative to the ultrarelativistic particle velocity, are radiated in a crystal of any length, but the entire PX characteristics arise in a crystal with length satisfying the condition

$$
\omega L\left|g_{00}^{\prime}\right| \gtrsim 1 \text {. }
$$

These features are of importance for a use of PX as an X-ray source, or for investigations of crystal structure. In the X-ray spectral range the inequality (29) means that $L \gtrsim 10^{4} \mathrm{~cm}$, and later on, we shall consider this case. Then the angle integration in equation (18) may be carried out analytically using

$$
\left|\frac{1-\exp \left(i \omega L q_{1 s}^{\tau}\right)}{q_{1 s}^{\tau}}\right|^{2} \approx \pi \delta\left(\operatorname{Re} q_{1 s}^{\tau}\right) \frac{1-\exp \left[-2 \omega L \operatorname{Im} q_{1 s}^{\tau}\left(x_{0}\right)\right]}{\operatorname{Im} q_{1 s}^{\tau}\left(x_{0}\right)}
$$

where $\delta(y)$ is the Dirac $\delta$-function and $x_{0}$ is determined from the equality

$$
\operatorname{Re} q_{1 s}^{\tau}\left(x_{0}\right)=0 .
$$

The PX spectrum calculated by means of equations (18) and (30) is shown in figure $5 a$. From these results, it follows that the frequency of the radiation emitted along the direction of $\omega_{\tau} \mathbf{v}+\tau$ equals $\omega_{\tau}$ with a spectral spread

$$
\Delta \omega=\omega_{\tau} \sqrt{\left|g_{00}\left(\omega_{\tau}\right)\right|+m^{2} / E^{2}} .
$$

Here,

$$
\omega_{\tau}=\frac{\tau^{2}}{2\left|\tau_{z}\right|}=\frac{\tau}{2 \sin \theta_{0} / 2}
$$

and this frequency varies continuously with a rotation of the crystal [15].

We remark that the energy resolution of modern X-ray detectors is not sufficient in order to investigate the shape of PX-lines. Therefore, let us consider the total number $N_{s}^{\tau}$ of the photons emitted in the Bragg direction. When the particle energy satisfies the condition

$$
E \gtrsim E_{0} \equiv m\left|g_{00}\left(\omega_{\tau}\right)\right|^{-1 / 2},
$$


and $\omega L g_{00}^{\prime \prime} \gg 1$, the value of $N_{\tau}$ is obtained as the following expression $[10,15]$ :

$$
\begin{gathered}
N_{s}^{\tau}=e^{2} \frac{\left|\tau_{\perp}^{2}-\tau_{Z}^{2}\right|}{8 \tau_{Z}^{2}} \mathfrak{J} \frac{\left|g_{10}^{(s)}\left(\omega_{\tau}\right)\right|^{2}}{g_{00}^{\prime \prime}\left(\omega_{\tau}\right)}\left|\ln \left\{\left(\frac{m^{2}}{E^{2}}+\delta_{s}-g_{00}\right)^{2}+\left|g_{10}^{(s)}\right|^{2}-\delta_{s}^{2}\right\}\right| \\
\delta_{s}=\frac{\operatorname{Re} \sqrt{r_{s}} \cdot \operatorname{Im} \sqrt{r_{s}}}{g_{00}^{\prime \prime}} ; \tau \neq 0 .
\end{gathered}
$$
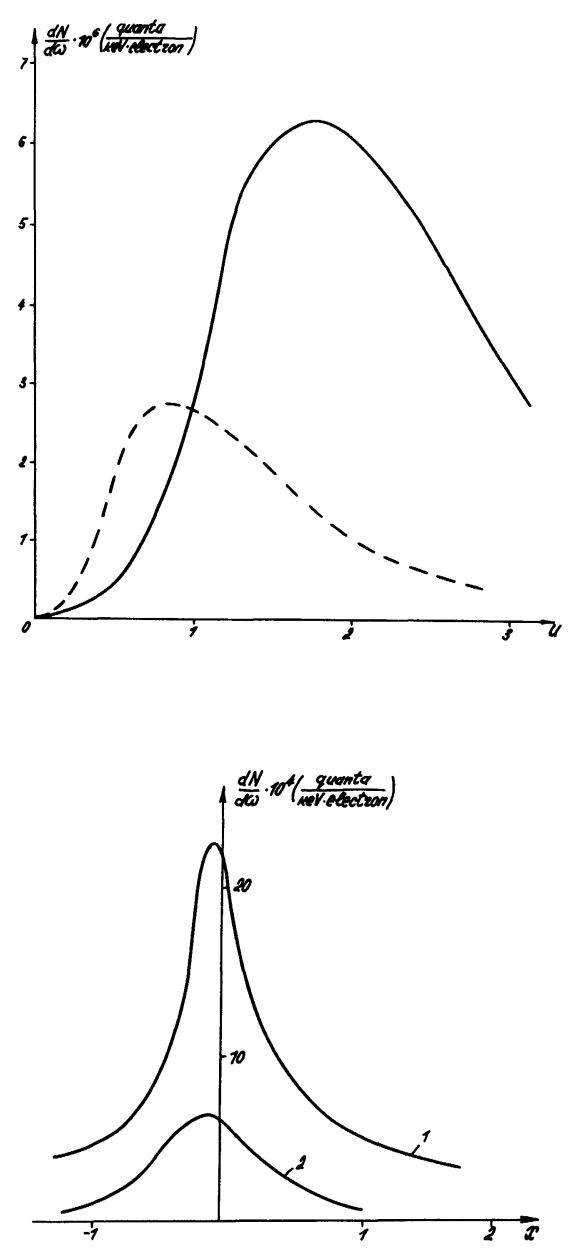

Fig. 5. - Distribution of frequency for parametric radiation $(E=1 \mathrm{GeV}) . a) \mathrm{X}$-rays in the non Mössbauer crystal ${ }^{56} \mathrm{Fe}$. $u=\frac{2 \omega \tau_{z}+\tau^{2}}{\omega^{2}} \times 10^{2.5} ; L=0.01 \mathrm{~cm},--\omega=2 \mathrm{keV}$; $\theta_{0}=35^{\circ} ;---\omega=30 \mathrm{keV} ; \theta_{0}=61^{\circ}$.

b) Resonant gamma-rays in Mössbauer crystals. $1-{ }^{57} \mathrm{Fe}$; $2-{ }^{183} \mathrm{~W} ; x=\frac{2}{\Gamma}\left(\omega-\omega_{r}\right) ; u_{r}=\frac{2 \omega_{r} \tau_{z}+\tau^{2}}{\omega_{r}^{2}}=3 \times 10^{-3}$.

For a silicon crystal it follows from equation (32) that

$$
E_{0} \approx 450 \mathrm{MeV} \text {, }
$$

and one can find from formula (33) the estimate

$$
N_{s m}^{\tau} \approx 2 \times 10^{-6} \text { photons/per electron. }
$$

If equation (32) is not satisfied the number of photons sharply decreases to the value

$$
N_{s}^{\tau} \approx\left(\frac{E}{E_{0}}\right)^{4} N_{s m}^{\tau} .
$$

In order to find the number of resonant $\gamma$-quanta $N_{\gamma}$ emitted in a Mössbauer crystal it is necessary to carry out the numerical integration in equation (18) because the approximation (30) is inapplicable in this case. Figure $5 b$ shows the results of such a calculation. In references $[10,15]$ an analytical formula was found which enables us to estimate $N_{\gamma}$ approximately. This formula is

$$
N_{\gamma} \approx e^{2} J \frac{g_{00}^{\prime \prime}\left(\omega_{r}\right)}{\left|\Delta^{\prime \prime}\right|^{1 / 2}}\left|g_{00}\left(\omega_{r}\right)\right|^{1 / 2} \frac{\Gamma}{\omega_{r}} ; \quad E>E_{0} .
$$

The $\gamma$-quanta angular spread is defined by the expression

$$
\Delta \theta_{\gamma}=\frac{\left|\Delta^{\prime \prime}\right|^{1 / 2}}{\sqrt{\left|g_{00}\left(\omega_{r}\right)\right|}} \ll \Delta \theta_{\tau}
$$

and is substantially less than that for X-rays.

For ${ }^{57} \mathrm{Fe}$ crystal $\left(\omega_{r}=14.4 \mathrm{keV}\right)$ and $\mathfrak{J}=1 \mathrm{~A}$ one obtains from equation (35) the result

$$
N_{\gamma} \approx 10^{6} \quad \text { quanta/per second }
$$

This intensity corresponds to the radiation from an isotropic source with activity of $10^{8}$ curies because of the small angular spread $\Delta \theta_{\gamma} \sim 10^{-6}$.

\section{Use of PX for gamma-laser pumping.}

In references $[14,15]$ a use of $P X$ for roentgenography and for obtaining monochromatic and focused X-ray beams and continuously tunable sources was considered. In the present paragraph we shall consider the efficiency of PX for gamma-laser pumping. The advantages of PX can be used most effectively in this particular problem [22]. They consist of high spectral intensity, small angular spread and the possibility of varying the radiation frequency in a continuous manner.

In accordance with reference [17] the maximum values of X-ray intensity for parametric (PX), channelling $(\mathrm{CH})$, and synchrotron $(S)$ radiations are related 
as follows

$$
\left(\frac{\mathrm{d} N}{\mathrm{~d} \omega}\right)_{\mathrm{PX}} \approx 10^{2}\left(\frac{\mathrm{d} N}{\mathrm{~d} \omega}\right)_{\mathrm{CH}} \approx 10^{4}\left(\frac{\mathrm{d} N}{\mathrm{~d} \omega}\right)_{S} .
$$

In order to avoid the damaging of the crystal by resonant nuclei it is necessary to use the radiation formed in another crystal for pumping (see Fig. 6). We shall also suppose that the pumping is operated via a three-level scheme shown in figure 7. Then the ratio of the number of excited nuclei $N^{\prime}$ formed for a time $\tau \approx \Gamma^{-1}$ to the quantity of unexcited nuclei $N$ is equal to

$$
\xi=\frac{N^{\prime}}{N} \approx N_{\gamma} \tau \frac{\sigma_{r}}{s}
$$

where $N_{\gamma}$ is the number of $\gamma$-quanta entering the Mössbauer crystal per unit time; $\sigma_{r}$ is the resonant absorption cross-section; $s$ is the crystal cross-sectional area.

The threshold of gamma-laser operation is achieved when $\xi \sim 1$ and using the equations (34) and (37) one can finds that the value of threshold current is defined by the formula [23]

$J_{\text {th }} \gtrsim \frac{s\left|\Delta^{\prime \prime}\right|^{1 / 2} \omega_{r}}{e^{2} g_{00}^{\prime \prime}\left(\omega_{r}\right)\left|g_{00}\left(\omega_{r}\right)\right|^{1 / 2} \sigma_{r}} \frac{\Gamma}{\Gamma_{1}} \times 10^{-9}(\mathrm{~A})$.

In the case of ${ }^{57} \mathrm{Fe}$ and $s=10^{-4} \mathrm{~cm}^{2} ; \Gamma / \Gamma_{1} \approx 10^{-3}$ one obtains

$$
J_{\text {th }} \gtrsim 10^{9} \mathrm{~A} \text {. }
$$

Such a current is practically unattainable but there are several ways to optimize the pumping scheme and decrease $\mathfrak{J}_{\mathrm{th}}$. In particular it is possible to operate the

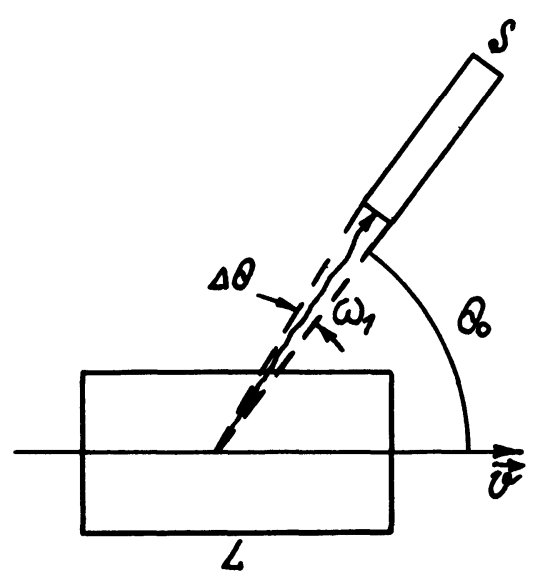

Fig. 6. - Crystal arrangement for gamma-laser pumping.

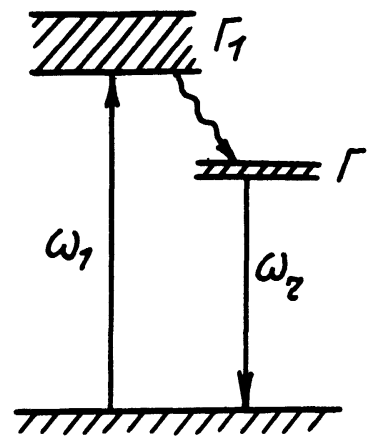

Fig. 7. - Diagram of resonant nucleus levels.

pumping scheme with polarized nuclei [24]. This decreases the threshold to

$$
J_{\text {th }} \gtrsim 10^{6} \mathrm{~A} \text {. }
$$

As shown above, the maximum value of the radiation coherent length is limited by the absorption length $\left(L_{\mathrm{a}}=\left(\omega \sqrt{\Delta^{\prime \prime}}\right)^{-1}\right.$ in the Mössbauer crystal). But let us suppose that the electron radiates in a crystal of needle form with a transverse size less than $L_{\mathrm{a}}$. Then PX, which travels in the direction of $\left(\omega_{r} \mathbf{v}+\right.$ $\tau)$, will be not absorbed in the crystal and the coherence length will be proportional to the length of the entire crystal. This decreases $J_{\text {th }}$ by two orders of magnitude if the crystal length were $1 \mathrm{~cm}$.

But the most important decrease in $\mathfrak{J}_{\text {th }}$ is possible if one uses PX from an electron beam modulated with frequency $\omega_{r}$. In this case the coherent radiation intensity will be proportional to $\mathfrak{J}^{2}$. The number of photons $N_{\gamma}^{\text {coh }}$ emitted in such a process is

$$
N_{\gamma}^{\mathrm{coh}}=\delta^{2} \jmath \tau \frac{1}{s\left(\omega_{r} \Delta \theta_{\gamma}\right)^{2}} N_{\gamma},
$$

where $N_{\gamma}$ is given by equation (34); $\delta$ is the beam modulation depth at the frequency $\omega_{r}$.

Electron beam modulation with frequency $\omega_{r}$ is a very difficult problem. It may be achieved for example, from a many-quanta interaction of the beam with high intensity optical electromagnetic waves [25]. Because of the small angular spread of PX, $N_{\gamma}^{\text {coh }}$ is substantially greater than $N_{\gamma}$ even for $\delta \ll 1$.

Thus if all of the above-mentioned improvements of the pumping were taken into account, then for a gamma-laser using ${ }^{57} \mathrm{Fe}$ one can could have

$$
J_{\mathrm{th}} \approx 10^{-1} \mathrm{~A}
$$

when $\delta=10^{-2}$ and the electron energy is $E \gtrsim 1 \mathrm{GeV}$.

Such a value of the current is attained by modern accelerators. 


\section{References}

[1] KaPITSA, S. P., Uspekhy Fiz. Nauk 129 (1979) 549.

[2] Alfuorov, D. F., Bashmakov, Yu. A., Bessonov, E. G., Zh. Technich. Fiz. 43 (1973) 2126.

[3] Olsen, H., Maximon, L., Phys. Rev. 114 (1959) 887.

[4] Baryshevsky, V. G., Dubovskaya, I. YA., Doklady Akademii Nauk USSR 231 (1976) 1335.

[5] Kumakhov, M. A., Phys. Letters A59 (1976) 413.

[6] Fainberg, Ya. B., Thiznjak, N. A., Zh. Exper. Teor. Fiz. 32 (1957) 883.

[7] Ter-Mikaeljan, M. L., Influence of Medium on Electromagnetic Processes by High Energy, Erevan (Akad. Nauk Arm. USSR) 1969.

[8] Baryshevsky, V. G., Doklady Akad. Nauk BSSR 15 (1971) 306.

[9] Baryshevsky, V. G., Feranchuk, I. D., Zh. Exper. Teor. Fiz. 61 (1971) 944 ; addendum, ibid 64 (1973) 760.

[10] Baryshevsky, V. G., Feranchuk, I. D., Doklady Akad. Nauk BSSR 18 (1974) 499; Phys. Letters A 57 (1976) 183.

[11] Garibjan, G. M., Jan SHEE, Zh. Exper. Teor. Fiz. 63 (1972) 1198.

[12] Fjodorov, V. V., Smirnov, A. I., Zh. Exper. Teor. Fiz. Pisma 23 (1976) 34.
[13] Mendlowitz, H., Glass, J., Phys. Letters A 73 (1979) 363.

[14] Baryshevsky, V. G., Feranchuk, I. D., Phys. Letters A 76 (1980) 452; USSR Patent (1974) № 482834

[15] Feranchuk, I. D., Crystallographija 24 (1979) 289.

[16] Akhiezer, A. I., Berestetsky, V. B., Quantum Electrodynamics (Nauka) 1969.

[17] FeranchuK, I. D., Zh. Tekhnich. Fiz. 49 (1979) 1552.

[18] Afanas'ev, A. M., Kagan, Yu. M., Zh. Exper. Teor. Fiz. 48 (1965) 327.

[19] Batterman, B., Cole, H., Rev. Mod. Phys. 36 (1964) 681.

[20] Feranchuk, I. D., Izvestija Akad. Nauk USSR Fiz. (1981) 109.

[21] Kolpakov, A. V., Yadernaya Fiz. 16 (1972) 1003.

[22] Baldwin, G. C., Solem, J. C., Gol'dansky, V. I., Rev. Mod. Phys. 53 (1981) 687.

[23] Baryshevsky, V. G., FeranchuK, I. D., Transactions XI Confer. Charged Particles Collisions in Crystals, Moscow State University (1982) 213.

[24] Vysotsky, V. I., Zh. Exper. Teor. Fiz. 77 (1979) 492. [25] Avetissjan, G. K., Zh. Tekhnich. Fiz. 50 (1980) 1857. 